\title{
Indeterminate Colitis - Update on Treatment Options
}

\author{
Niranjani Venkateswaran' \\ Scott Weismiller' \\ Kofi Clarke $\mathbb{1}^{2}$ \\ 'Division of General Internal Medicine, \\ Department of Medicine, Pennsylvania \\ State University College of Medicine, \\ Hershey, PA, USA; ${ }^{2}$ Division of \\ Gastroenterology and Hepatology, \\ Department of Medicine, Pennsylvania \\ State University College of Medicine, \\ Hershey, PA, USA
}

\begin{abstract}
Indeterminate colitis (IC) is described in approximately 5-15\% of patients with inflammatory bowel disease (IBD). It usually reflects a difficulty or lack of clarity in distinguishing between ulcerative colitis (UC) and Crohn's disease (CD) on biopsy or colectomy specimens. The diagnostic difficulty may explain the variability in the reported prevalence and incidence of IC. Clinically, most IC patients tend to evolve over time to a definite diagnosis of either UC or CD. IC has also been interchangeably described as inflammatory bowel disease unclassified (IBDU). This review offers an overview of the available limited literature on the conventional medical and surgical treatments for IC. In contrast to the numerous studies on the medical management of $\mathrm{UC}$ and $\mathrm{CD}$, there are very few data from dedicated controlled trials on the treatment of IC. The natural evolution of IC more closely mimics UC. Regarding medical options for treatment, most patients diagnosed with IC are treated similarly to UC, and treatment choices are based on disease severity. Others are managed similarly to $\mathrm{CD}$ if there are features suggestive of $\mathrm{CD}$, including fissures, skin tags, or rectal sparing. In medically refractory IC, surgical treatment options are limited and include total proctocolectomy (TPC) and ileal pouch-anal anastomosis (IPAA), with its associated risk factors and complications. Post-surgical complications and pouch failure rates were historically thought to be more common in IC patients, but recent meta-analyses reveal similar rates between UC and IC patients. Future therapies in IBD are focused on known mechanisms in the disease pathways of UC and CD. Owing to the lack of IC-specific studies, clinicians have traditionally and historically extrapolated the data to IC patients based on their symptomatology, clinical course, and endoscopic findings.
\end{abstract}

Keywords: indeterminate colitis, medical treatment, ulcerative colitis, Crohn's disease, total proctocolectomy, ileal pouch-anal anastomosis

\section{Introduction}

Inflammatory bowel disease (IBD) consists of a spectrum of immune-mediated bowel diseases that usually manifest in genetically predisposed individuals. Although primarily a luminal bowel disease with two defined ends of the spectrum Crohn's disease (CD) and ulcerative colitis (UC) - there are multiple extraintestinal manifestations (EIMs) and a variant described as indeterminate colitis (IC). IC has also been referred to as inflammatory bowel disease unclassified (IBDU).

In this review, we use the term IC for consistency to refer to IC/IBDU.

The distinction between $\mathrm{CD}$ and UC is important and could have significant implications on the choice, type, and timing of disease-related surgery, disease course, prognosis, and medical treatment. ${ }^{1} \mathrm{UC}$ is characterized by superficial inflammation involving the rectum and extending proximally in a variable, diffuse, and typically
Division of Gastroenterology an

Hepatology, Department of Medicine,

Pennsylvania State University College of

Medicine, 500 University Drive, Hershey,

PA, 17033, USA

Tel $+|7| 7-53|-874|$

$\mathrm{Fax}+1$ 7|7-53I-6770

Email kclarke@pennstatehealth.psu.edu 
continuous distribution. $\mathrm{CD}$ is characterized by transmural inflammation in any part of the gastrointestinal tract with intervening normal areas, and is typically rectal sparing. IC may have characteristics of both $\mathrm{CD}$ and UC, often with eventual differentiation over the course of time. A few older studies have examined the proportion of patients evolving from IC to either CD or UC. In the study by Meucci et al, $80 \%$ of IC patients eventually acquired a definitive diagnosis of $\mathrm{UC}$ or $\mathrm{CD}$, within 8 years of diagnosis. ${ }^{2}$

Indeterminate colitis (IC) was proposed as a provisional classification prior to establishing a definitive diagnosis of either $\mathrm{CD}$ or $\mathrm{UC}$, and represents a subtype of IBD involving mucosal inflammation with clinical and pathological features that are not easily distinguished. ${ }^{3,4}$

The term inflammatory bowel disease unclassified (IBDU) has been used interchangeably with IC, specifically defined as IBD with no definitive features of CD or UC. It was introduced by Kent et al in instances where there were inadequate clinical data or histopathological examination showing some features of $\mathrm{CD}$ and other changes indistinguishable from UC. ${ }^{5}$ In 2005, the International Classification of Diseases, 10th revision (ICD-10) included IC as a diagnosis, separate from $\mathrm{CD}$ and UC. In the clinical setting, it is not uncommon to have patients initially labeled as having IC eventually evolve to UC or CD.

This review article provides a comprehensive review of IC, with a primary focus on its medical and surgical treatment. The available data are predominantly from studies in adult patients, which focused on patients with UC and $\mathrm{CD}$ with relatively small cohorts of IC patients included in the trials. We opted to include data from the occasional references in the pediatric literature owing to limited studies on IC in adult cohorts. In instances where we reference the pediatric literature, we offer our thoughts and discuss limitations on generalizability of the data. In addition, we briefly discuss the management of complications associated with IC, surgical management and its complications, and future therapeutic options.

Our thoughts on each section are highlighted at the end of the section.

\section{Epidemiology, Diagnosis, and Clinical Features of Indeterminate Colitis}

The prevalence rate of IC is estimated to be $22 / 100,000$, and it has a higher incidence in females and Caucasian patients. ${ }^{6}$
Several previous studies have shown that a diagnosis of IC is present in $5-15 \%$ of IBD cases. ${ }^{1,3}$ However, the diagnosis can be highly variable among institutions and individual pathologists owing to diagnostic difficulties. ${ }^{7,8}$ The frequency of establishing a diagnosis is dependent on the awareness of the broad spectrum of pathology seen in UC and CD. The classic endoscopic and histological features of UC or CD do not always conform to the traditional findings, which can lead to the diagnosis of IC. ${ }^{9}$ The location and distribution of the disease are important in differentiating UC from CD. Sequential biopsy changes in the mucosa of UC can evolve with time, leading to variation in the extent of involvement over time and a lack of an endoscopic-histological correlation. In quiescent disease, only minimal histological changes are found, making a histological differentiation of $\mathrm{CD}$ and UC difficult. Backwash ileitis can also present with a mild degree of inflammation in the distal ileum. ${ }^{9}$ Significant inflammation in such settings in the terminal ileum may raise the possibility of a diagnosis of $\mathrm{CD}$. There also appears to be a lack of consistency and clarity among pathologists regarding the definition of IC. A previous study evaluated the biopsy results of pathologists from 24 medical centers on colonic specimens from IBD patients, followed by an experienced IBD pathologist's review. The expert review resulted in a changed diagnosis in $45 \%$ of the specimens. ${ }^{10}$ Common causes for uncertainty in IBD pathology are fulminant colitis, insufficient endoscopic specimens, and failure to incorporate and utilize the major diagnostic features for $\mathrm{CD}$ and UC. ${ }^{11}$

Compared to UC, patients with IC have a younger age of onset and have more extensive and severe disease. The male to female ratio is usually close to 1 . IC patients tend to use immunosuppressive medications more frequently, and have a higher risk of colorectal cancer, colectomy, and pouch failure compared to UC patients. ${ }^{12}$ In addition, a family history of IBD is more common in IC compared to both $\mathrm{UC}$ and $\mathrm{CD}$. Associated EIMs are similar and equally common in IC patients compared to UC and CD. ${ }^{13}$

It is important for clinicians to provide a relevant and concise history to pathologists in patients with suspected IC to help to establish a diagnosis. If needed and clinically relevant, a second opinion from an expert gastrointestinal pathologist should be sought.

\section{Medical Treatment of Indeterminate Colitis}

There is insufficient evidence on specific medical management of IC owing to a lack of large randomized prospective 
treatment trials. This may also be partly due to the uncertain diagnosis and relatively small numbers of patients. Thus, patients with IC are typically treated similarly to UC patients based on the clinical disease severity, as well as the extent and severity of endoscopic and histological findings. ${ }^{12}$ Some clinical trials of adult patients with IBD have included small numbers of patients with IC, but have not specifically addressed treatment response in IC patients as a subgroup. The spectrum of conventional treatments previously studied includes corticosteroids, thiopurines (azathioprine or mercaptopurine), aminosalicylates (eg, 5-aminosalicylic acid [5-ASA]), calcineurin inhibitors, biologics, and small molecules.

Conventional treatments and advances in the treatment paradigm of IC are discussed in the next sections and are summarized in Table 1.

The discussion includes the use of these medications in UC and CD as a segue into their use in IC. In each of the sections on treatment options, we review and summarize the available literature on the use of each agent across the spectrum of IBD and opine on the specific use in IC.

\section{5-Aminosalicylic Acid Preparations}

Aminosalicylates such as 5-ASA remain the first line therapy for both induction and maintenance of mild to moderate $\mathrm{UC}$, but have not been shown to be particularly effective in the management of CD. The exception is sulfasalazine, which is superior to placebo in colonic CD.

The proposed mechanisms of action of aminosalicylates include increased expression of peroxisome proliferator-activated receptors in the gastrointestinal epithelial cells, which results in anti-inflammatory properties. They also decrease colonic inflammation by inhibiting the cyclo-oxygenase enzymes. ${ }^{14}$ Both oral and rectal 5-ASA therapies are effective in inducing and maintaining remission in UC. ${ }^{15}$ Sulfasalazine was the first developed aminosalicylate and was shown to be highly effective for the treatment of UC, with some efficacy in colonic CD. However, it is not well tolerated, which was a restrictive factor for optimizing dosage and led to the development of newer formulations. ${ }^{16}$ There is insufficient evidence regarding differences in effectiveness between the different oral 5-ASA formulations. ${ }^{17}$ In patients with mild to moderate UC, low- and standard-dose mesalamine has been shown to be effective for the induction and maintenance of remission. ${ }^{18}$ A meta-analysis of 15 studies indicated that the use of mesalamine and probiotics together had improved clinical efficacy in patients with UC. ${ }^{19}$ The magnitude of benefit was small when using high-dose mesalamine compared with standard dosage, and it was associated with higher costs. ${ }^{18}$

Based on these studies, 5-ASAs were extended to treat patients with mild IC, especially with features more suggestive of UC. Therapy usually includes 5-ASA suppositories for proctitis and proctosigmoiditis. Adequate doses of 5-ASA or sulfasalazine (3.6-4.8 g/day for 5-ASA and 4-6 g/day for sulfasalazine) were used for moderately active disease. ${ }^{20}$

It is worth noting that there are insufficient dedicated trial data to support the efficacy of these medications in IC. However, in clinical practice, 5-ASAs have been used in IC, ostensibly based on the extrapolation of data from trials in UC and CD.

Table I Different Classes of Medical Treatment for Indeterminate Colitis

\begin{tabular}{|l|l|l|l|l|}
\hline Therapy & $\begin{array}{l}\text { Strength of } \\
\text { Evidence }\end{array}$ & $\begin{array}{l}\text { First Line: Yes/ } \\
\text { No }\end{array}$ & $\begin{array}{l}\text { Risk of Side Effects: Low, Intermediate, } \\
\text { High }\end{array}$ & Disease Severity \\
\hline Anti-TNF-alpha agents & Inadequate & No & Intermediate & $\begin{array}{l}\text { Severe and } \\
\text { refractory }\end{array}$ \\
\hline $\begin{array}{l}\text { Thiopurines: azathioprine/ } \\
\text { 6-MP }\end{array}$ & Inadequate & No & Intermediate & Moderate \\
\hline Steroids & Moderate & No & High & $\begin{array}{l}\text { Moderate to } \\
\text { severe }\end{array}$ \\
\hline Vedolizumab & Inadequate & Unknown & Low & Unknown \\
\hline Ustekinumab & Inadequate & Unknown & Low & Unknown \\
\hline Mesalamines & Moderate & Yes & Low & Mild \\
\hline
\end{tabular}

Notes: We reviewed all available literature using the following databases: PubMed, Medscape, and UpToDate. Owing to the scarce data, IC has been looked at as a subgroup in larger IBD studies. 


\section{Corticosteroids}

Steroids are effective in both luminal UC and CD for inducing remission. Budesonide is most commonly used in patients with $\mathrm{CD}$ because of its activity in the distal ileum and right colon. ${ }^{21}$ Other steroids, including hydrocortisone and prednisolone, are commonly used and were previously the mainstay of IBD treatment. However, steroids have no role in maintaining remission in either UC or $\mathrm{CD}^{22}$ Patients are usually classified as "steroid responsive", "steroid dependent", or "steroid resistant". Longterm steroid use is associated with significant toxicities, including weight gain, increased risk of diabetes mellitus, adrenal suppression, bone loss, eye disease, and hypertension. These side effects, in combination with the known lack of efficacy for steroids as maintenance therapy in IBD, have resulted in the development and more frequent use of immunomodulatory therapies. ${ }^{22}$ One study showed that an early response to intravenous steroids and maintenance therapy with biologics was associated with a lower rate of relapse in severe $\mathrm{UC}^{23}$

The role of steroids is limited to controlling symptoms in flares in IBD, as a bridge to more definitive therapy, or the induction of remission. Their use has been extended similarly to include patients diagnosed with IC.

\section{Thiopurines}

Thiopurines have been shown to maintain remission in both UC and $\mathrm{CD}^{21}$ Previous studies have investigated the use of thiopurine independent of concurrent antitumor necrosis factor (anti-TNF) therapy. ${ }^{24}$ The available data call into question the overall benefits of thiopurine monotherapy in patients with IBD, especially when balanced against the known risks, which include lymphomas. There is some benefit in patients with perianal disease. Both azathioprine (AZA) and 6-mercaptopurine (6-MP) have been used in moderate to severe IBD as monotherapy or as part of combination therapy in patients with steroid-dependent disease. ${ }^{21}$ They can cause myelosuppression, especially in patients with low levels of thiopurine methyltransferase (TPMT) enzyme activity. It is standard clinical practice to check the TPMT levels in patients with IBD contemplating thiopurine therapy. Pancreatitis is one of the contraindications for continued use of these agents. ${ }^{21}$ There is a slight increase in the risk of development of non-Hodgkin's lymphoma and nonmelanoma skin cancer. ${ }^{17}$
In our opinion, these side effects and the scanty available literature limit the utility of thiopurines as monotherapy in patients with IC. Furthermore, there are other available therapies with more data on their efficacy in IC patients.

\section{Methotrexate}

Methotrexate (MTX) is a folate analog which interferes with DNA synthesis and has anti-inflammatory effects. It has been used in both $\mathrm{UC}$ and $\mathrm{CD} .{ }^{14}$ McDonald et al conducted a systemic review of large randomized trials, which suggested that MTX provided a benefit for induction of remission and complete withdrawal from steroids in refractory $\mathrm{CD} .{ }^{25}$ Although several retrospective studies have shown favorable effects of MTX in UC, ${ }^{26,27}$ two randomized controlled trials did not show any difference in either induction or maintenance. ${ }^{28,29}$ Overall, based on the meta-analysis and randomized studies, MTX monotherapy is not recommended for induction therapy. ${ }^{30}$ The use of MTX in IC has been studied in the pediatric population. Assa et al conducted a retrospective study which showed that IBDU patients had similar outcomes to CD patients at final follow-up in terms of the efficacy, tolerance, and safety of MTX. ${ }^{31}$ MTX was also recommended for use in children with IC who are intolerant or refractory to AZA/6-MP. Hepatotoxicity, which presents with elevated aminotransferase levels and significant risk of teratogenicity in pregnancy, remains a concern associated with MTX. Somewhat reassuringly, liver fibrosis is uncommon. ${ }^{32}$

To the best of our knowledge, there are no convincing data on the use of MTX as monotherapy in IC.

\section{Calcineurin Inhibitors}

Navazo et al showed that oral microemulsion cyclosporine was an effective drug in the initial management of patients with UC and IC flare unresponsive to steroids. ${ }^{33}$ Cyclosporine has been used to treat both moderate and severe UC. One small randomized placebo-controlled study reported efficacy in using cyclosporine in steroidrefractory UC in hospitalized patients. ${ }^{34}$ Another randomized trial from Japan showed higher rates of mucosal healing with tacrolimus but low clinical remission rates. ${ }^{35}$ More recently, clinicians have favored using infliximab as rescue therapy in hospitalized patients with acute severe UC, perhaps because of concerns over the narrow therapeutic window for cyclosporine. ${ }^{36}$

The narrow therapeutic window for cyclosporine, including potential renal toxicity, may have contributed 
to infliximab being favored in severely active $\mathrm{UC}$ and refractory IC as well.

\section{Vedolizumab}

Vedolizumab is a second generation humanized antiadhesion medication that blocks the interaction between $\alpha_{4} \beta_{7}$-integrin and MadCAM-1. ${ }^{37}$ The GEMINI trials I and II were randomized, placebo-controlled studies that compared vedolizumab to placebo in patients with $\mathrm{UC}$ and $\mathrm{CD}$, respectively.

In GEMINI I, UC patients were randomized to either placebo or vedolizumab at weeks 0 and 2 . At week 6 , the response rates were $47.1 \%$ and $25.5 \%$ among patients in the vedolizumab and placebo groups, respectively $(p<0.001)$. The clinical remission rates were $16.9 \%$ in the active group and $5.4 \%$ in the control group. There was also a significant difference in mucosal healing between the active and control placebo groups ( $40.9 \%$ vs $24.8 \%)$. Responders following induction were re-randomized to receive vedolizumab every 8 weeks (Q8W) or every 4 weeks (Q4W), or placebo, and followed up to week 52. For the maintenance phase of the trial, the clinical remission rates at week 52 were $41.8 \%$ and $44.8 \%$ for patients assigned to vedolizumab $\mathrm{Q} 8 \mathrm{~W}$ and $\mathrm{Q} 4 \mathrm{~W}$, respectively, compared with $15.9 \%$ for placebo patients. Results from the maintenance therapy showed that durable clinical response, durable clinical remission, mucosal healing, and glucocorticoid-free remission at week 52 were all higher among patients assigned to vedolizumab regimens compared to the placebo group. ${ }^{38}$

In GEMINI II, patients with active CD were randomized to either vedolizumab or placebo at weeks 0 and 2 . During the induction phase, clinical remission rates were $14.5 \%$ and $6.8 \%$ for the vedolizumab and placebo groups, respectively, at week $6(p=0.02)$. Responders from the induction phase were re-randomized to receive vedolizumab Q8W or Q4W, or placebo, for the maintenance phase of the trial. At week 52, the clinical remission rates were $39 \%, 36.4 \%$, and $21.6 \%$ for patients who received vedolizumab Q8W and $\mathrm{Q} 4 \mathrm{~W}$, and the placebo group, respectively. ${ }^{39}$

The VISIBLE I trial compared the efficacy of subcutaneous/intravenous vedolizumab to a placebo group. The results showed statistical significance, with clinical remission following both subcutaneous and intravenous infusions. ${ }^{40}$ Another trial compared vedolizumab to adalimumab, and showed higher rates of endoscopic improvement $(39.7 \%$ vs $27.7 \%)$, and clinical $(31.3 \%$ vs $22.5 \%)$ and histopathological remission $(10.4 \%$ vs $3.1 \%)$ in patients who received vedolizumab at week $52 .{ }^{41}$ It is worth noting that none of these trials reported an increase in the rate of infections. However, there was a mild increase in the risk of infusion reactions and malignancy in less than $5 \%$ of the patients. ${ }^{38,39,41}$

To date and to the best of our knowledge, there have been no studies specifically evaluating the use of vedolizumab exclusively in patients with IC. Their use in patients in IC is based on extrapolated data from studies on patients with $\mathrm{UC}$ and $\mathrm{CD}$.

\section{Ustekinumab}

Ustekinumab is a monoclonal immunoglobulin $\mathrm{G}(\mathrm{IgG})$ antibody that targets the p40 subunit of the inflammatory cytokines interleukin-12 (IL-12) and IL-23. ${ }^{37}$ Two identical randomized placebo-controlled trials evaluated the efficacy of ustekinumab in moderate to severe CD patients: UNITI-1 and UNITI-2. Patients who either had a primary or secondary non-response to TNF or had adverse effects were included in the UNITI-1 trial, while patients who either failed or had severe side effects from conventional treatment but were largely anti-TNF therapy naïve were included in the UNITI-2 trial. In both trials, patients received one of two doses of ustekinumab or placebo. Both trials showed higher clinical response rates at week 6 with both doses of ustekinumab compared to the placebo group. Patients who responded were included in the IMUNITI trial for maintenance therapy with ustekinumab for either Q8W or Q12W. In both groups, the clinical remission rates were $53.1 \%$ and $48.8 \%$ in patients who received ustekinumab Q8W and Q12W, respectively, and 35.9\% in the placebo group $(p=0.04) .{ }^{42}$

The UNIFI trial evaluated the efficacy of ustekinumab as 8 -week induction and 44-week maintenance therapy in patients with moderate to severe UC. The rate of clinical remission was higher among patients who received ustekinumab compared to the placebo group ( $15.5 \%$ vs $5.3 \%$, $p<0.001)$. Patients who responded in the induction phase underwent second randomization and received maintenance therapy with ustekinumab Q12W or Q8W, or placebo. The clinical remission rate at week 44 was significantly higher among patients who received $90 \mathrm{mg}$ subcutaneous ustekinumab Q12W (38.4\%) and ustekinumab Q8W (43.8\%) compared with the placebo group (24.0\%). The UNIFI trial showed that there were significant benefits in clinical remission, endoscopic improvement, and reduction in fecal calprotectin in patients with 
UC who received ustekinumab. ${ }^{43}$ Another study also evaluated the role of ustekinumab as a rescue treatment option for refractory moderate to severe UC. ${ }^{44}$

Although these studies indicate the efficacy and durability of response of ustekinumab in both $\mathrm{UC}$ and $\mathrm{CD}$ patients with areassuring safety profile, there are inadequate data available on the efficacy of ustekinumab in IC. By extension of the data in $\mathrm{UC}$ and $\mathrm{CD}$, we opine that it could be offered as atherapeutic option to the appropriate patient after discussion as part of shared decision making.

\section{Treatment of Refractory Indeterminate Colitis Infliximab}

Infliximab, a chimeric monoclonal $\operatorname{IgG}_{1}$ antibody directed against human TNF-alpha, has been explored as a potential therapy for medically refractory IC. Papadakis et al evaluated infliximab in a cohort of 20 patients with IC who had steroid-resistant or steroid-dependent colitis. All patients had failed 5-ASA, 14 were refractory to thiopurines, and three had failed cyclosporine therapy. Fourteen patients $(70 \%)$ showed a complete clinical response, defined as the cessation of colitis-related symptoms (abdominal cramping, diarrhea, and bleeding), two patients $(10 \%)$ showed a partial response, defined by a reduction in the same symptoms, while four $(20 \%)$ showed no response. ${ }^{45}$ Herrlinger et al evaluated infliximab salvage therapy in 24 patients with either steroidrefractory or steroid-dependent disease who had failed induction with tacrolimus treatment. They noted that six of 24 tacrolimus-resistant patients (17\%) achieved clinical remission after infliximab infusion, while an additional four $(17 \%)$ had an initial response but ultimately required colectomy. Furthermore, 14 of 24 (58\%) had no response and required colectomy. ${ }^{46}$

Based on the results of these few, small studies, the utility of infliximab in medically refractory IC is inconclusive but could be considered after weighing the risks and benefits of treatment versus surgical intervention.

\section{Total Proctocolectomy and Ileal Pouch- Anal Anastomosis}

Total proctocolectomy (TPC) and ileal pouch-anal anastomosis (IPAA), first reported in 1933, is now part of the spectrum of standard surgical treatment of UC patients who are acutely non-responsive to medical therapy, chronically refractory to medical treatment, or steroid dependent with adverse side effects, or in whom neoplastic transformation has occurred. The study by $\mathrm{Yu}$ et al suggests that approximately $15 \%$ of patients with IC undergoing TPC and IPAA are reclassified as CD on long-term follow-up. It is reasonable to infer that the natural history of IC tends to overlap more with UC. ${ }^{47}$ In addition, the data lend some comfort in offering surgical intervention as a potential treatment option for IC patients. However, IC patients who undergo IPAA appear to be more likely than UC patients to have complications, including pouch failure. ${ }^{9,47-50}$

While the earliest studies evaluating pouch complications showed a significantly increased risk of pouch failure in IC compared to UC patients, more recent data contrast with these findings. One retrospective study noted that after surgical classification as UC or IC, there was no significant difference in the incidence of acute pouchitis, chronic pouchitis, or de novo CD between UC and IC groups. ${ }^{51}$ Numerous other studies noted similar pouch failure rates between IC and UC. ${ }^{52-54}$ Another retrospective case-matched analysis found that post-IPAA Crohn's disease rates were significantly increased in IC patients, although pouch failure rates were similar. ${ }^{55}$ An additional analysis of IC patients showed high rates of pouchitis (57\%) among both CD-like and non-CD disease-like behavior, with the $\mathrm{CD}$-like patients requiring more medications ( $95 \%$ vs $18 \%$ ), dilations ( $41 \%$ vs $0 \%$ ), and pouch reoperations (32\% vs $6 \%$ ) compared to the non-CD group. ${ }^{56}$ Of note, there was no difference in the Pouch Function Score between the two groups, which contrasts with the poor results previously published for patients with $\mathrm{CD}$ undergoing IPAA. ${ }^{57,58}$

To further clarify the outcomes of IPAA in patients with IC, a meta-analysis published in 2020, including 17 studies of IPAA outcomes, found a weighted mean pouch failure rate of $7.5 \%$ and weighted mean pouch complication rate of $67 \%$ in patients with IC. Patients with IC and UC had similar rates of pouch failure, pouchitis, anastomotic leak, stricture, and small bowel obstruction. IC patients had significantly higher odds of developing pouch fistula, pelvic sepsis, pelvic or cuff abscess, perineal complications, and an ultimate diagnosis of $\mathrm{CD}$ compared to their UC counterparts. ${ }^{59}$

Given the relatively suboptimal results in patients with IC who eventually evolve into CD, IBD serological tests have been explored to help differentiate between $\mathrm{CD}$ and UC. These tests include perinuclear anti-neutrophil cytoplasmic antibody (pANCA), anti-Saccharomyces 
cerevisiae antibody (ASCA), anti-outer membrane porin $\mathrm{C}$ antibody (anti-OmpC), bacterial flagellin antibody (anticBIR-1), and Pseudomonas fluorescens-related protein antibody (anti-I2). It is worth noting that these tests have been studied in patients with defined UC and CD, and not extensively studied in IC patients. A prospective study of 97 IBD patients using pANCA and ASCA as potential differentiators revealed that $50 \%$ of patients with IC were negative for both markers, and the remaining patients tested positive on one or both tests; Furthermore, more CD patients showedASCA positive, ANCA negative whiles UC patients showed ASCA negative, ANCA positive.. ${ }^{60}$ ASCA and pANCA have shown sensitivity rates of only 40-60\% when differentiating between $\mathrm{UC}$ and $\mathrm{CD}$, limiting their utility in practice. Another observational study of IC patients over 12 months showed that a positive pANCA was associated with a likelihood ratio of 1.4 for a subsequent diagnosis of UC at 1 year, but neither ASCA nor anti-OmpC was associated with a subsequent diagnosis of $\mathrm{CD}^{61}$ Additional larger, multicenter prospective studies are needed to explore the predictive value of IBD serology in patients with IC evolving into UC or CD.

Based on the current limited evidence, it is reasonable to offer patients IPAA for IC as long as they are informed of the operative risks and potential pouch complications, including CD-like disease.

\section{Management of Complications Associated with Indeterminate Colitis}

IC symptoms include abdominal pain, abdominal cramping, persistent diarrhea, blood in the stool, rectal bleeding, weight loss, reduced appetite, fatigue, and changes in bowel patterns. Management involves a constellation of analgesics, medical therapy, and surgical options, depending on the response to treatment and disease severity. Providers typically refrain from using non-steroidal antiinflammatory drugs (NSAIDs), because of their association with IBD flares, and opioids, because of challenges associated with long-term use. Other options include antidepressants, anticonvulsants, acetaminophen, and local therapy, depending on patient-specific factors.

Some complications arise from surgery, as in patients undergoing TPC with IPAA. A meta-analysis of 13 studies comparing outcomes of pouch surgery between patients with UC and IC found that UC patients developed complications of pouchitis $(24.4 \%)$, small bowel obstruction
(7.4\%), anastomotic structure (4.9\%), pouch fistula $(2.7 \%)$, pelvic sepsis $(2.5 \%)$, perineal complications $(1.9 \%)$, pelvic or cuff abscess $(1.4 \%)$, and an ultimate diagnosis of $\mathrm{CD}(0.67 \%)$. It also found that compared with UC patients, IC patients had higher odds of developing pouch fistula, pelvic sepsis, pelvic or cuff abscess, perineal complications, and an ultimate diagnosis of $\mathrm{CD}{ }^{59}$

Pouchitis is the most common complication following IPAA. Symptoms typically involve increased stool frequency, increased defecation urgency, pelvic pain, or bowel incontinence. Primary prevention of pouchitis typically involves avoidance of NSAIDs, given their higher association with chronic pouchitis. ${ }^{62}$ Initial treatment usually consists of 2 weeks of ciprofloxacin, despite only a few studies exploring antibiotic efficacy, with other options including metronidazole or tinidazole. ${ }^{63}$ Chronic pouchitis may develop in patients who are unresponsive to antibiotics or in whom they are ineffective. In those instances, considering other potential etiologies, including atypical infection and immune-mediated diseases, requiring the addition of 5-ASA, steroids, and vedolizumab or ustekinumab, is an alternative approach. ${ }^{64,65}$ Probiotics have been examined in a few, small trials, which failed to show any significant benefit from probiotics alone, but they may provide an improvement in symptoms or remission in patients who had previously been treated with antibiotics. ${ }^{66}$ Surgical management, including redo or takedown of the pouch, is considered in medicationrefractory patients whose quality of life has been significantly affected by symptomatology.

Small bowel obstruction can be initially treated with nasogastric decompression (NGD). If NGD fails, then surgical management could be considered.

Anastomotic pouch strictures can result in bacterial overgrowth, pouch dilatation, defecatory challenges, and bowel obstruction. Management ranges from benign procedures of evaluation under anesthesia (EUA) and dilatation to endoscopic balloon/needle knife therapy and medical therapy, with the most severe treatment options including stricturoplasty, proximal diversion and stricture resection, or pouch excision. ${ }^{67}$

Fistula formation is a more common complication of CD but can still occur after IPAA. Fistulas may be pouchvaginal, pouch-vesical, or pouch-anal fistulas. Pouchvaginal fistulas can be treated conservatively with seton placement and fistula plugs, but owing to the poor success rates, surgical intervention is typically required. Pouchvesical or pouch-anal fistulas can typically be treated with 
serial EUA, abscess drainage, and sphincter-preserving surgery. ${ }^{66}$ There is emerging evidence that stem cell therapy may be useful in the treatment of fistulas. A metaanalysis analyzing 29 studies found a higher rate of fistula healing with stem cell therapy compared to placebo, with higher healing rates in perianal and transsphincteric fistulas compared to rectovaginal fistulas. ${ }^{68}$

Based on the available data, similar approaches to the treatment of complications in patients with UC and CD can be applied to patients with IC in the appropriate clinical setting.

\section{Extraintestinal Manifestations}

IBD can involve multiple organ systems, including the joints, skin, biliary tract, and eyes in $25-40 \%$ of IBD patients. $^{69}$ These EIMs are more common in CD than UC, and in adults more so than children, but have not been well defined in IC. ${ }^{70}$ Other EIMs and associated conditions include pyoderma gangrenosum, erythema, nodosum, Sweet syndrome, psoriasis, atopic dermatitis, hidradenitis suppurativa, axial and peripheral spondyloarthropathies, dactylitis, enthesitis, primary sclerosing cholangitis, uveitis, and episcleritis. ${ }^{71}$ Two systematic reviews exploring the diagnosis and treatment of EIMs confirmed not only that anti-TNF agents were the primary treatment for EIMs, but also that they were effective in achieving a clinical response in over $50 \%$ of cases, except for primary sclerosing cholangitis. ${ }^{72,73}$ Vedolizumab was not found to be efficacious in treating pre-existing EIMs but could have potential use for reducing the incidence of new EIMs. Minimal data exist for ustekinumab and tofacitinib.

There are also no clear guidelines available for the evaluation and follow-up for each EIM, and this should be studied further in patients with IC. Future large multicenter studies or registry-derived data are required.

\section{Evaluation and Treatment of Associated Conditions, Age-Appropriate Screening, and Preventive Care}

\section{Infections and Vaccinations}

IBD patients have an increased risk of infection not only due to their disease, but also due to the immunosuppressive therapies necessary for disease control. Therefore, age-appropriate vaccination should be discussed at the time of diagnosis. The risk of gastrointestinal infection is higher among IBD patients compared to control groups, with studies showing that enteric infections were responsible for $10.5 \%$ of all IBD relapses. ${ }^{74,75}$ Clostridium difficile is the most common cause of infection and has been shown to increase the risk of colectomy, postoperative infectious complications, and mortality. ${ }^{76,77}$ It is also important to check for cytomegalovirus (CMV) superinfection in moderate to severe colitis flares, specifically in those with corticosteroid-refractory disease or receiving immunomodulatory therapy. ${ }^{78}$ A meta-analysis by Ford and Peyrin-Biroulet found that $0.9 \%$ of 4135 patients on anti-TNF therapy in randomized controlled trials developed an opportunistic infection, with a two-fold increased risk of infection with Mycobacterium tuberculosis (TB), herpes simplex and zoster, oral/esophageal candidiasis, CMV, Epstein-Barr virus (EBV), and Nocardia. ${ }^{79}$

In the absence of data specific to IC, age-appropriate vaccinations should remain a key aspect of treatment in all patients with IBD, including IC. In addition, testing and pretreatment screening for hepatitis $\mathrm{B}$ and $\mathrm{C}$ (HBV, HCV), human immunodeficiency virus (HIV), and TB infection is vital.

\section{Osteoporosis and Osteopenia}

Both CD and UC patients are at increased risk for bone disease. This is thought to be due to active inflammatory states resulting in increased bone resorption, calcium and vitamin D malabsorption/deficiency, as well as prolonged steroid use. Estimated rates of osteopenia and osteoporosis are reported as $35 \%$ and $15 \%$ of patients, respectively. ${ }^{80-82}$ Bone density should be assessed prior to initiation of glucocorticoid therapy, as well as counseling for exercise and lifestyle modifications, including smoking cessation. Treatment with vitamin D and calcium should be initiated in appropriate clinical settings. Patients with osteopenia and significantly increased risk of fractures or prior fractures, as well as frank osteopenia, should be comanaged with endocrinology and/or rheumatology.

We recommend a similar approach in patients with IC.

\section{Vascular Disease}

IC patients are at a higher risk of cardiovascular disease given the nature of the systemic inflammation. The study by Paschou et al found a decrease in insulin levels and insulin resistance in non-obese, non-diabetic patients who received biological therapy. ${ }^{83}$

We recommend assessing and controlling factors related to cardiovascular disease in all IBD patients. 


\section{Cancer Screening}

Historically, IC patients have been excluded from population-based analyses of cancer risk calculations in IBD patients. UC patients have an elevated risk of colon cancer and should therefore begin screening colonoscopy 8 years after disease onset. In patients with associated primary sclerosing cholangitis, screening colonoscopies should begin at diagnosis and yearly thereafter.

In patients with IC, clinicians should consider a similar approach to UC patients described above, keeping in mindthat there are no strong data specific to the IC cohort.

\section{Future Therapeutic Options}

Patients with IC are managed similarly to those diagnosed with UC, including both medical regimens and surgery for those who are non-responsive to medication. Newer therapies showing some efficacy, including drugs targeting IL-12, IL-23, and the janus kinase/signal transducer and activator of transcription proteins (JAK/STAT) pathway, as well as sphingosine-1-phosphate receptor modulators (S1P), have been approved or are in development for the management of IBD.

IL-12 and IL-23, both of which contain a p40 subunit in their overall structure, have become therapeutic targets owing to their downstream effects on TNF and T1 cell differentiation/ IL-17 secretion, respectively. ${ }^{84}$ These include ustekinumab, as mentioned in an earlier subsection, and brazikumab. In a phase IIa study, brazikumab, which selectively inhibits IL-23, showed clinical improvement in CD patients who had failed anti-TNF therapy in $49 \%$ of 119 patients with moderate to severe $\mathrm{CD}{ }^{85}$ Mirikizumab, guselkumab, and tildrakizumab are all currently being studied and may be useful for both $\mathrm{UC}$ and $\mathrm{CD}$, pending further exploration.

The most well-known JAK/STAT therapy, tofacitinib, mainly targets JAK1/JAK3 and has shown to be effective in UC through the OCTAVE trials. ${ }^{86,87}$ These trials were phase III, randomized, double-blind, placebo-controlled trials of tofacitinib therapy in moderate to severe UC patients after failure with conventional/anti-TNF therapy, eventually showing effectiveness for induction and maintenance therapy compared to placebo. In the OCTAVE Induction 1 and 2 trials, the primary endpoint was remission at 8 weeks, while in the OCTAVE Sustain trials the primary endpoint was remission at 52 weeks. The most common reported side effects were nasopharyngitis, arthralgia, and headache, which seemed to occur at similar rates in both drug and control groups. Other more concerning adverse events noted included thrombosis, herpes infection, and dosedependent dyslipidemia.

Indicators from ongoing trials with filgotinib and upadacitinib (JAK1 inhibitors) show favorable results regarding symptom improvement, clinical remission, and histological evaluation. Filgotinib was tested in the FITZROY study, a phase II, randomized, double-blind, placebo-controlled trial, which found clinical remission in patients with moderate to severe $\mathrm{CD}^{88}$ The primary endpoint of clinical remission was defined as a Crohn's Disease Activity Index (CDAI) of less than 150 at week 10. Although side effects were similar between the study and control groups, serious infections occurred in the treatment but not the control group. Filgotinib was also evaluated in SELECTION, a phase IIb/ III, randomized, double-blind, placebo-controlled trial that found efficaciousness for the induction and maintenance of clinical remission in moderate to severe UC compared to placebo. ${ }^{89}$ The primary endpoint was clinical remission by Mayo endoscopic criteria, rectal bleeding, and stool frequency scores at 10 and 58 weeks. Adverse events were similar between the treatment groups in all three studies and the control group, with infection leading all other events.

The U-ACCOMPLISH trial, a phase III, randomized, double-blind, placebo-controlled trial for upadacitinib, confirmed clinical, endoscopic, and histological improvement in moderate to severe UC patients, as shown in the U-ACHIEVE trial, but the data have yet to be published. The primary endpoint was clinical remission via the Adapted Mayo Score. The most common side effects were acne, increased blood creatinine phosphokinase, and anemia, but overall safety findings were consistent with previous studies of the drug. The CELEST trial, a phase II, randomized, double-blind, placebo-controlled trial for patients with moderate to severe $\mathrm{CD}$ with inadequate response to immunosuppressants or TNF-alpha inhibitors, found endoscopic, but not clinical remission. ${ }^{39}$

Sphingosine-1-phosphate receptor modulators are effective by inactivating lymphocytes before they are able to leave lymphoid tissue, preventing them from reaching their end-organ targets. The TOUCHSTONE study was a phase II, randomized, double-blind, placebocontrolled trial with ozanimod for moderate to severe UC, with a primary endpoint of clinical remission (Mayo Clinic Score $\leq 2)$ at 8 weeks. In this trial, ozanimod was more effective than placebo for induction of clinical remission, and it has been approved for clinical use in UC. ${ }^{90}$ 
Further advances in therapy will likely rely on the accuracy of diagnosis of IC, with possible identification of certain biomarkers that could predict subsets of patients and future disease behavior. Importantly, randomized controlled trials involving IC patients are needed to provide more definitive answers and guidance for effective therapeutic options. In the absence of any data, we cannot opine on the utility of any of the newer molecules in the treatment of IC.

\section{Pediatric Population}

IC is twice as common in pediatric populations compared to their adult counterparts, with as many as $10 \%$ of children receiving IC as an initial diagnosis. ${ }^{91}$ Similarly to adults, rates of IC diagnosis decrease over time. As in adults, treatment of UC and CD has well-developed guidelines, but no guidelines exist for IC in pediatric populations. One retrospective, multicenter analysis analyzing 23 centers of pediatric IBD found that the most common first line treatment for active IC was mesalamine. Other treatments, including thiopurines, methotrexate, cyclosporine, and infliximab, were not clearly defined but were generally used based on clinical response to 5 -ASA. ${ }^{92}$

\section{Disclosure}

The authors report no relevant conflicts of interest related to this review.

\section{References}

1. Maher MM. Inflammatory bowel disease: review and future view. Front Biosci. 2012;4:1638-1647. doi:10.2741/e485

2. Meucci G, Bortoli A, Riccioli FA, et al. Frequency and clinical evolution of indeterminate colitis: a retrospective multi-centre study in northern Italy. GSMII (Gruppo di Studio per le Malattie Infiammatorie Intestinali). Eur J Gastroenterol Hepatol. 1999;11 (8):909-913. doi:10.1097/00042737-199908000-00018

3. Geboes K, Colombel JF, Greenstein A, et al. Indeterminate colitis: a review of the concept-what's in a name? Inflamm Bowel Dis. 2008;14(6):850-857. doi:10.1002/ibd.20361

4. Price AB. Overlap in the spectrum of non-specific inflammatory bowel disease-"colitis indeterminate". J Clin Pathol. 1978;31(6):567-577. doi:10.1136/jcp.31.6.567

5. Kent TH, Ammon RK, DenBesten L. Differentiation of ulcerative colitis and regional enteritis of colon. Arch Pathol. 1970;89(1):20-29.

6. Emad Mansoor FJ-D, Cheema T, Saleh MA, Regueiro M, Katz J, Cooper GS. Epidemiology of indeterminate colitis in the United States between 2014 and 2019: a Population-Based National Study. Am J Gastroenterol. 2019;114(p):S1594. doi:10.14309/01. ajg.0000601220.52856.c5

7. Yantiss RK, Odze RD. Diagnostic difficulties in inflammatory bowel disease pathology. Histopathology. 2006;48(2):116-132. doi:10.1111/ j.1365-2559.2005.02248.x
8. Tremaine WJ. Is indeterminate colitis determinable? Curr Gastroenterol Rep. 2012;14(2):162-165. doi:10.1007/s11894-0120244-x

9. Guindi M, Riddell RH. Indeterminate colitis. J Clin Pathol. 2004;57 (12):1233-1244. doi:10.1136/jcp.2003.015214

10. Farmer M, Petras RE, Hunt LE, Janosky JE, Galandiuk S. The importance of diagnostic accuracy in colonic inflammatory bowel disease. Am J Gastroenterol. 2000;95(11):3184-3188. doi:10.1111/ j.1572-0241.2000.03199.x

11. Odze RD. A contemporary and critical appraisal of "indeterminate colitis". Mod Pathol. 2015;28(Suppl 1):S30-46. doi:10.1038/ modpathol.2014.131

12. Geboes K, De Hertogh G. Indeterminate colitis. Inflamm Bowel Dis. 2003;9(5):324-331. doi:10.1097/00054725-200309000-00007

13. Rudolph WG, Uthoff SM, McAuliffe TL, Goode ET, Petras RE, Galandiuk S. Indeterminate colitis: the real story. Dis Colon Rectum. 2002;45(11):1528-1534. doi:10.1007/s10350-004-6461-0

14. Kozuch PL, Hanauer SB. Treatment of inflammatory bowel disease: a review of medical therapy. World $J$ Gastroenterol. 2008;14 (3):354-377. doi:10.3748/wjg.14.354

15. Engel MA, Neurath MF. New pathophysiological insights and modern treatment of IBD. $J$ Gastroenterol. 2010;45(6):571-583. doi:10.1007/s00535-010-0219-3

16. Karagozian R, Burakoff R. The role of mesalamine in the treatment of ulcerative colitis. Ther Clin Risk Manag. 2007;3(5):893-903.

17. Bernstein CN. Treatment of IBD: where we are and where we are going. Am J Gastroenterol. 2015;110(1):114-126. doi:10.1038/ ajg. 2014.357

18. Singh S, Feuerstein JD, Binion DG, Tremaine WJ. AGA technical review on the management of mild-to-moderate ulcerative colitis. Gastroenterology. 2019;156(3):769-808 e729. doi:10.1053/j. gastro.2018.12.008

19. Tian C, Huang Y, Wu X, Xu C, Bu H, Wang $\mathrm{H}$. The efficacy and safety of mesalamine and probiotics in mild-to-moderate ulcerative colitis: a systematic review and meta-analysis. Evid Based Complement Alternat Med. 2020;2020:6923609. doi:10.1155/2020/6923609

20. Burakoff R. Indeterminate colitis: clinical spectrum of disease. $J$ Clin Gastroenterol. 2004;38(5 Suppl 1):S41-43. doi:10.1097/01. mcg.0000123991.13937.7e

21. Pithadia AB, Jain S. Treatment of inflammatory bowel disease (IBD). Pharmacol Rep. 2011;63(3):629-642. doi:10.1016/S1734-1140(11) 70575-8

22. Ford AC, Bernstein CN, Khan KJ, et al. Glucocorticosteroid therapy in inflammatory bowel disease: systematic review and meta-analysis. Am J Gastroenterol. 2011;106(4):590-599; quiz 600. doi:10.1038/ ajg. 2011.70

23. Salameh R, Kirchgesner J, Allez M, et al. Long-term outcome of patients with acute severe ulcerative colitis responding to intravenous steroids. Aliment Pharmacol Ther. 2020;51(11):1096-1104. doi:10.1111/apt.15751

24. Cosnes J, Bourrier A, Laharie D, et al. Early administration of azathioprine vs conventional management of Crohn's Disease: a randomized controlled trial. Gastroenterology. 2013;145(4):758765e752; quiz e714-755. doi:10.1053/j.gastro.2013.04.048

25. McDonald JW, Tsoulis DJ, Macdonald JK, Feagan BG. Methotrexate for induction of remission in refractory Crohn's disease. Cochrane Database Syst Rev. 2012;12:CD003459. doi:10.1002/14651858. CD003459.pub3

26. Fraser AG, Morton D, McGovern D, Travis S, Jewell DP. The efficacy of methotrexate for maintaining remission in inflammatory bowel disease. Aliment Pharmacol Ther. 2002;16(4):693-697. doi:10.1046/j.1365-2036.2002.01227.x

27. Cummings JR, Herrlinger KR, Travis SP, Gorard DA, McIntyre AS, Jewell DP. Oral methotrexate in ulcerative colitis. Aliment Pharmacol Ther. 2005;21(4):385-389. doi:10.1111/j.1365-2036.2005.02331.x 
28. Mate-Jimenez J, Hermida C, Cantero-Perona J, Moreno-Otero R. 6-mercaptopurine or methotrexate added to prednisone induces and maintains remission in steroid-dependent inflammatory bowel disease. Eur J Gastroenterol Hepatol. 2000;12(11):1227-1233. doi:10.1097/00042737-200012110-00010

29. Oren R, Arber N, Odes S, et al. Methotrexate in chronic active ulcerative colitis: a double-blind, randomized, Israeli multicenter trial. Gastroenterology. 1996;110(5):1416-1421. doi:10.1053/ gast.1996.v110.pm8613046

30. Heron V, Loftus EV Jr. Nonbiologic immune suppression in ulcerative colitis. Gastroenterol Clin North Am. 2020;49(4):731-738. doi:10.1016/j.gtc.2020.07.003

31. Assa A, Hartman C, Weiss B, et al. Long-term outcome of tumor necrosis factor alpha antagonist's treatment in pediatric Crohn's disease. $J$ Crohns Colitis. 2013;7(5):369-376. doi:10.1016/j. crohns.2012.03.006

32. Hannoodee M, Mittal M. Methotrexate. In: StatPearls. Treasure Island (FL); 2021.

33. Navazo L, Salata H, Morales S, et al. Oral microemulsion cyclosporine in the treatment of steroid-refractory attacks of ulcerative and indeterminate colitis. Scand J Gastroenterol. 2009;36(6):610-614. doi:10.1080/003655201750163051

34. Lichtiger S, Present DH, Kornbluth A, et al. Cyclosporine in severe ulcerative colitis refractory to steroid therapy. $N$ Engl $\mathrm{J}$ Med. 1994;330(26):1841-1845. doi:10.1056/NEJM199406303302601

35. Ogata $\mathrm{H}$, Matsui T, Nakamura M, Hirai F, et al. A randomized dose finding study of oral tacrolimus (FK506) therapy in the management of hospitalized patients with refractory ulcerative colitis. Inflamm Bowel Dis. 2012;18:803-808. doi:10.1002/ibd.21853

36. Jarnerot G, Hertervig E, Friis-Liby I, et al. Infliximab as rescue therapy in severe to moderately severe ulcerative colitis: a randomized, placebo-controlled study. Gastroenterology. 2005;128 (7):1805-1811. doi:10.1053/j.gastro.2005.03.003

37. Sedano R, Almradi A, Ma C, Jairath V, Feagan BG. Novel therapeutics for the treatment of ibd: current status and future directions. Curr Treat Options Gastroenterol. 2020;18:442-461. doi:10.1007/s11938020-00299-7

38. Feagan BG, Rutgeerts P, Sands BE, et al. Vedolizumab as induction and maintenance therapy for ulcerative colitis. $N$ Engl $J$ Med. 2013;369(8):699-710. doi:10.1056/NEJMoa1215734

39. Sandborn WJ, Feagan BG, Rutgeerts P, et al. Vedolizumab as induction and maintenance therapy for Crohn's disease. $N$ Engl J Med. 2013;369(8):711-721. doi:10.1056/NEJMoa1215739

40. Sandborn WJ, Baert F, Danese S, et al. Efficacy and safety of vedolizumab subcutaneous formulation in a randomized trial of patients with ulcerative colitis. Gastroenterology. 2020;158(3):562572 e512. doi:10.1053/j.gastro.2019.08.027

41. Sands BE, Schreiber S, Lirio RA. Vedolizumab versus adalimumab for moderate-to-severe ulcerative colitis. reply. $N$ Engl $J$ Med. 2020;382(1):93-94. doi:10.1056/NEJMc1915739

42. Feagan BG, Sandborn WJ, Gasink C, et al. Ustekinumab as induction and maintenance therapy for crohn's disease. $N$ Engl $\mathrm{J} \mathrm{Med}$. 2016;375(20):1946-1960. doi:10.1056/NEJMoa1602773

43. Sands BE, Sandborn WJ, Panaccione R, et al. Ustekinumab as induction and maintenance therapy for ulcerative colitis. $N$ Engl J Med. 2019;381(13):1201-1214. doi:10.1056/NEJMoa1900750

44. Ochsenkuhn T, Tillack C, Szokodi D, et al. Clinical Outcomes with ustekinumab as rescue treatment in therapy-refractory or therapy-intolerant ulcerative colitis. United Eur Gastroenterol J. 2019;8(1):91-98. doi:10.1177/2050640619895361

45. Papadakis KA, Treyzon L, Abreu MT, Fleshner PR, Targan SR, Vasiliauskas EA. Infliximab in the treatment of medically refractory indeterminate colitis. Aliment Pharmacol Ther. 2003;18(7):741-747. doi:10.1046/j.1365-2036.2003.01739.x
46. Herrlinger KR, Barthel DN, Schmidt KJ, et al. Infliximab as rescue medication for patients with severe ulcerative/indeterminate colitis refractory to tacrolimus. Aliment Pharmacol Ther. 2010;31 (9):1036-1041. doi:10.1111/j.1365-2036.2010.04267.x

47. Yu CS, Pemberton JH, Larson D. Ileal pouch-anal anastomosis in patients with indeterminate colitis: long-term results. Dis Colon Rectum. 2000;43(11):1487-1496. doi:10.1007/BF02236726

48. McIntyre PB, Pemberton JH, Wolff BG, Dozois RR, Beart RW Jr. Indeterminate colitis. Long-term outcome in patients after ileal pouch-anal anastomosis. Dis Colon Rectum. 1995;38(1):51-54. doi:10.1007/BF02053857

49. Atkinson KG, Owen DA, Wankling G. Restorative proctocolectomy and indeterminate colitis. Am J Surg. 1994;167(5):516-518. doi:10.1016/0002-9610(94)90248-8

50. Koltun WA, Schoetz DJ Jr, Roberts PL, Murray JJ, Coller JA, Veidenheimer MC. Indeterminate colitis predisposes to perineal complications after ileal pouch-anal anastomosis. Dis Colon Rectum. 1991;34(10):857-860. doi:10.1007/BF02049696

51. Murrell ZA, Melmed GY, Ippoliti A, et al. A prospective evaluation of the long-term outcome of ileal pouch-anal anastomosis in patients with inflammatory bowel disease-unclassified and indeterminate colitis. Dis Colon Rectum. 2009;52(5):872-878. doi:10.1007/ DCR.0b013e31819f5d4c

52. Brown CJ, MacLean AR, Cohen Z, Macrae HM, O'Connir B, McLeod RS. Crohn's disease and indeterminate colitis and the ileal pouch-anal anastomosis: outcomes and patterns of failure. Dis Colon Rectum. 2005;48(8):1542-1549. doi:10.1007/s10350-005-0059-z

53. Pishori T, Dinnewitzer A, Zmora O, et al. Outcome of patients with indeterminate colitis undergoing a double-stapled ileal pouch-anal anastomosis. Dis Colon Rectum. 2004;47(5):717-721. doi:10.1007/ s10350-003-0116-4

54. Dayton MT, Larsen KR, Christiansen DD. Similar functional results and complications after ileal pouch-anal anastomosis in patients with indeterminate vs ulcerative colitis. Arch Surg. 2002;137(6):690-694; discussion 694-695. doi:10.1001/archsurg.137.6.690

55. Jackson KL, Stocchi L, Duraes L, Rencuzogullari A, Bennett AE, Remzi FH. Long-term outcomes in indeterminate colitis patients undergoing ileal pouch-anal anastomosis: function, quality of life, and complications. $J$ Gastrointest Surg. 2017;21(1):56-61. doi:10.1007/s11605-016-3306-9

56. Netz U, Galbraith NJ, O'Brien S, et al. Long-term outcomes following ileal pouch-anal anastomosis in patients with indeterminate colitis. Surgery. 2018;163(3):535-541. doi:10.1016/j. surg.2017.11.014

57. Sagar PM, Dozois RR, Wolff BG. Long-term results of ileal pouchanal anastomosis in patients with Crohn's disease. Dis Colon Rectum. 1996;39(8):893-898. doi:10.1007/BF02053988

58. Lightner AL, Mathis KL, Dozois EJ, et al. Results at up to 30 years after ileal pouch-anal anastomosis for chronic ulcerative colitis. Inflamm Bowel Dis. 2017;23(5):781-790. doi:10.1097/ MIB.0000000000001061

59. Emile SH, Gilshtein H, Wexner SD. Outcome of ileal pouch-anal anastomosis in patients with indeterminate colitis: a systematic review and meta-analysis. J Crohns Colitis. 2020;14(7):1010-1020. doi:10.1093/ecco-jcc/jjaa002

60. Joossens S, Reinisch W, Vermeire S, et al. The value of serologic markers in indeterminate colitis: a prospective follow-up study. Gastroenterology. 2002;122(5):1242-1247. doi:10.1053/gast.2002.32980

61. Sura SP, Ahmed A, Cheifetz AS, Moss AC. Characteristics of inflammatory bowel disease serology in patients with indeterminate colitis. J Clin Gastroenterol. 2014;48(4):351-355. doi:10.1097/MCG.0000 00000000083

62. Achkar JP, Al-Haddad M, Lashner B, et al. Differentiating risk factors for acute and chronic pouchitis. Clin Gastroenterol Hepatol. 2005;3(1):60-66. doi:10.1016/S1542-3565(04)00604-4 
63. Nguyen N, Zhang B, Holubar SD, Pardi DS, Singh S. Treatment and prevention of pouchitis after ileal pouch-anal anastomosis for chronic ulcerative colitis. Cochrane Database Syst Rev. 2019;11:CD001176. doi:10.1002/14651858.CD001176.pub5

64. Ribaldone DG, Pellicano R, Saracco GM, Morino M, Astegiano M. Vedolizumab for treatment of chronic refractory pouchitis: a systematic review with pool analysis. Rev Esp Enferm Dig. 2020;112(1):59-63. doi:10.17235/reed.2019.6336/2019

65. Ollech JE, Rubin DT, Glick L, et al. Ustekinumab is effective for the treatment of chronic antibiotic-refractory pouchitis. Dig Dis Sci. 2019;64(12):3596-3601. doi:10.1007/s10620-019-05697-1

66. Mimura T, Rizzello F, Helwig U, et al. Once daily high dose probiotic therapy (VSL\#3) for maintaining remission in recurrent or refractory pouchitis. Gut. 2004;53(1):108-114. doi:10.1136/gut.53.1.108

67. Lucha PA Jr, Fticsar JE, Francis MJ. The strictured anastomosis: successful treatment by corticosteroid injections-report of three cases and review of the literature. Dis Colon Rectum. 2005;48 (4):862-865. doi:10.1007/s10350-004-0838-y

68. Cao Y, Su Q, Zhang B, Shen F, Li S. Efficacy of stem cells therapy for Crohn's fistula: a meta-analysis and systematic review. Stem Cell Res Ther. 2021;12(1):32. doi:10.1186/s13287-020-02095-7

69. Vavricka SR, Rogler G, Gantenbein C, et al. Chronological order of appearance of extraintestinal manifestations relative to the time of ibd diagnosis in the Swiss inflammatory bowel disease cohort. Inflamm Bowel Dis. 2015;21(8):1794-1800. doi:10.1097/MIB.0000000000000429

70. Kirsch R, Pentecost M, Hall Pde M, Epstein DP, Watermeyer G, Friederich PW. Role of colonoscopic biopsy in distinguishing between Crohn's disease and intestinal tuberculosis. J Clin Pathol. 2006;59(8):840-844. doi:10.1136/jcp.2005.032383

71. Ott C, Scholmerich J. Extraintestinal manifestations and complications in IBD. Nat Rev Gastroenterol Hepatol. 2013;10(10):585-595. doi:10.1038/nrgastro.2013.117

72. Guillo L, D'Amico F, Serrero M, et al. Assessment of extraintestinal manifestations in inflammatory bowel diseases: a systematic review and a proposed guide for clinical trials. United Eur Gastroenterol J. 2020;8(9):1013-1030. doi:10.1177/2050640620950093

73. Peyrin-Biroulet L, Van Assche G, Gomez-Ulloa D, et al. Systematic review of tumor necrosis factor antagonists in extraintestinal manifestations in inflammatory bowel disease. Clin Gastroenterol Hepatol. 2017;15(1):25-36 e27. doi:10.1016/j.cgh.2016.06.025

74. Goodhand JR, Alazawi W, Rampton DS. Systematic review: clostridium difficile and inflammatory bowel disease. Aliment Pharmacol Ther. 2011;33(4):428-441. doi:10.1111/j.1365-2036.2010.04548.x

75. Mylonaki M, Langmead L, Pantes A, Johnson F, Rampton DS. Enteric infection in relapse of inflammatory bowel disease: importance of microbiological examination of stool. Eur J Gastroenterol Hepatol. 2004;16(8):775-778. doi:10.1097/01.meg.0000131040.38607.09

76. Ananthakrishnan AN, McGinley EL, Saeian K, Binion DG. Temporal trends in disease outcomes related to Clostridium difficile infection in patients with inflammatory bowel disease. Inflamm Bowel Dis. 2011;17(4):976-983. doi:10.1002/ibd.21457

77. Navaneethan U, Mukewar S, Venkatesh PG, Lopez R, Shen B. Clostridium difficile infection is associated with worse long term outcome in patients with ulcerative colitis. J Crohns Colitis. 2012;6 (3):330-336. doi:10.1016/j.crohns.2011.09.005

78. McCurdy JD, Jones A, Enders FT, et al. A model for identifying cytomegalovirus in patients with inflammatory bowel disease. Clin Gastroenterol Hepatol. 2015;13(1):131-137; quiz e137. doi:10.1016/j.cgh.2014.05.026
79. Ford AC, Peyrin-Biroulet L. Opportunistic infections with anti-tumor necrosis factor-alpha therapy in inflammatory bowel disease: meta-analysis of randomized controlled trials. Am J Gastroenterol. 2013;108(8):1268-1276. doi:10.1038/ajg.2013.138

80. Agrawal M, Arora S, Li J, et al. Bone, inflammation, and inflammatory bowel disease. Curr Osteoporos Rep. 2011;9(4):251-257. doi:10.1007/s11914-011-0077-9

81. Targownik LE, Bernstein CN, Leslie WD. Risk factors and management of osteoporosis in inflammatory bowel disease. Curr Opin Gastroenterol. 2014;30(2):168-174. doi:10.1097/MOG.0000000 000000037

82. Briot K, Geusens P, Em Bultink I, Lems WF, Roux C. Inflammatory diseases and bone fragility. Osteoporos Int. 2017;28(12):3301-3314. doi:10.1007/s00198-017-4189-7

83. Paschou SA, Kothonas F, Lafkas A, et al. Favorable effect of anti-TNF therapy on insulin sensitivity in nonobese, nondiabetic patients with inflammatory bowel disease. Int $J$ Endocrinol. 2018;2018:6712901. doi:10.1155/2018/6712901

84. Baker KF, Isaacs JD. Novel therapies for immune-mediated inflammatory diseases: what can we learn from their use in rheumatoid arthritis, spondyloarthritis, systemic lupus erythematosus, psoriasis, Crohn's disease and ulcerative colitis? Ann Rheum Dis. 2018;77 (2):175-187. doi:10.1136/annrheumdis-2017-211555

85. Sands BE, Chen J, Feagan BG, et al. Efficacy and safety of MEDI2070, an antibody against interleukin 23, in patients with moderate to severe crohn's disease: a Phase 2a Study. Gastroenterology. 2017;153(1):77-86 e76. doi:10.1053/j. gastro.2017.03.049

86. Sandborn WJ, Su C, Sands BE, et al. Tofacitinib as induction and maintenance therapy for ulcerative colitis. $N$ Engl J Med. 2017;376 (18):1723-1736. doi:10.1056/NEJMoa1606910

87. Panes J, Vermeire S, Lindsay JO, et al. Tofacitinib in patients with ulcerative colitis: health-related quality of life in phase 3 randomised controlled induction and maintenance studies. J Crohns Colitis. 2018;12(2):145-156. doi:10.1093/ecco-jcc/jjx133

88. Vermeire S, Schreiber S, Petryka R, et al. Clinical remission in patients with moderate-to-severe Crohn's disease treated with filgotinib (the FITZROY study): results from a phase 2, double-blind, randomised, placebo-controlled trial. Lancet. 2017;389 (10066):266-275. doi:10.1016/S0140-6736(16)32537-5

89. Feagan BG, Danese S, Loftus EV Jr, et al. Filgotinib as induction and maintenance therapy for ulcerative colitis (SELECTION): a phase $2 \mathrm{~b} /$ 3 double-blind, randomised, placebo-controlled trial. Lancet. 2021;397(10292):2372-2384. doi:10.1016/S0140-6736(21)00666-8

90. Sandborn WJ, Feagan BG, Wolf DC, et al. Ozanimod induction and maintenance treatment for ulcerative colitis. $N$ Engl J Med. 2016;374 (18):1754-1762. doi:10.1056/NEJMoa1513248

91. Prenzel F, Uhlig HH. Frequency of indeterminate colitis in children and adults with IBD - a metaanalysis. J Crohns Colitis. 2009;3 (4):277-281. doi:10.1016/j.crohns.2009.07.001

92. Aloi M, Birimberg-Schwartz L, Buderus S, et al. Treatment options and outcomes of pediatric ibdu compared with other IBD subtypes: a retrospective multicenter study from the IBD porto group of ESPGHAN. Inflamm Bowel Dis. 2016;22(6):1378-1383. doi:10.1097/MIB.0000000000000767 


\section{Publish your work in this journal}

The Journal of Inflammation Research is an international, peerreviewed open-access journal that welcomes laboratory and clinical findings on the molecular basis, cell biology and pharmacology of inflammation including original research, reviews, symposium reports, hypothesis formation and commentaries on: acute/chronic inflammation; mediators of inflammation; cellular processes; molecular mechanisms; pharmacology and novel anti-inflammatory drugs; clinical conditions involving inflammation. The manuscript management system is completely online and includes a very quick and fair peerreview system. Visit http://www.dovepress.com/testimonials.php to read real quotes from published authors. 